\title{
Design, Synthesis and Biological Evaluation of Some Novel Chalcone-Sulfonamide Hybrids
}

\author{
MAHAMMADALI KHANUSIYA* and ZAKIRHUSEN GADHAWALA \\ Department of Chemistry, The HNSB Ltd Science College, Himatnagar, Gujarat, India \\ khanusiya.mali@gmail.com
}

Received 2 May 2018 / Accepted 3 July 2018

\begin{abstract}
A series of novel chalcone tethered sulphonamide hybrids 3a-5f were fabricated by condensing appropriate sulphonamide scaffold with methoxy and/or hydroxy substituted chalcones as a multi-target drug for therapeutic treatment. Chalcones were prepared by Claisen-Schmidt condensation of a substituted aldehyde with para aminoacetophenone. The synthesized chalconesulphonamide hybrids were screened by means of their antibacterial activity by NCCLS method. Among all these compounds, $\mathbf{5 e}$ and $\mathbf{5 c}$ were observed to displaying more potent growth inhibitory activity against bacterial strain and hybrid $\mathbf{5 a}$ shows more obvious antifungal activity. FT-IR, NMR and HR-LCMS spectral study assert the structures of synthesized sulphonamide - chalcone hybrids.
\end{abstract}

Keywords: Chalcones, Sulphonamide scaffold, Antibacterial, Antifungal

\section{Introduction}

Chalcone, an exceptional chemical template of two aromatic or heteroaryl rings tethered by a three-carbon $\alpha, \beta$-unsaturated carbonyl system, exhibiting a class of flavonoids. Flavonoids are inborn in fruits and vegetables. The plant containing chalcone derivatives are traditionally deputed for therapeutic concern. Chalcones were availed for their multifarious biological activities and there are a number of reviews that have dealt with the pharmacological and chemical bases of the biological activities noticed under the effect of chalcones. Natural and synthetic chalcones are greatly supportive to enhance numerous therapeutic activities ${ }^{1-10}$.

Numerous experimental activities on chalcone tantamount to the employment of three pointed strategies such as structural modification of both aryl rings, replacement of aryl ring with heteroaryl scaffold and molecular hybridization via conjugation with another potent pharmacophore for the enhancement of biological properties. Diverse substitutions on both aryl rings of chalcone, depending upon their position, induce to enhance their biological activities. Replacing aromatic by heteroaryl in chalcone also influences pharmacological activity. Chemically, chalcone hybrids are designed by linking chalcone derivative to another biologically potent scaffold such as sulphonamides, benzothiazoles; triazole, benzodiazepines, isoxazolines; imidazolones, indols, quinolines, coumarins etc., which have exhibited favorable biological activities ${ }^{11}$. 
Sulphonamides are referred as the compounds that have sulphonamide moiety $\left(-\mathrm{SO}_{2} \mathrm{NH}_{2}\right)$ in its structure and are found to possess various biologically interesting activities including antitumor activity. Sulpha drugs result in positive antibacterial and antifungal activities. Sulphadiazine, sulfamerazine and sulphadimidine are vital sulpha drugs used as a treatment against bacterial infections, cerebrospinal meningitis and allergic infections ${ }^{12}$. They are additive pharmaceutical drugs which are availed in numerous biological activities including antitumor $^{13}$, antibacterial ${ }^{14}$, antifungal ${ }^{15}$, anti-carbonic anhydrase ${ }^{16}$, diuretic $^{17}$, hypoglycemic $^{18}$, antithyroid $^{19}$ and anti $\mathrm{HIV}^{20}$. Anticancer activity of elected chalcone-sulphonamide hybrid (Figure 1) has been reported in many types of cancers including pancreatic, hepatic and colon. Structural activity relationship observation shows that chalcone containing sulphonamide group has a commendable effect on the biological activity ${ }^{21}$.<smiles>[R]NC1=CCC(C(=O)/C=C/c2ccc(Br)cc2)C=C1</smiles><smiles>O=C(/C=C/c1ccc(O)cc1)c1ccc(NS(=O)(=O)c2ccc(F)cc2)cc1</smiles>

Figure 1. Chalcone- sulphonamide hybrids

The biological activities of both pharmacophores point out to us to synthesize novel sulphonamide-chalcone hybrids which are known to be having such important microbial properties. Various aldehydes were chosen to synthesize substituted chalcones and these chalcones were condensed with biologically potent sulpha drugs which finally resulted in chalcone-sulphonamide hybrids.

\section{Experimental}

The starting materials and solvents were purchased from Sigma-Aldrich and SD Fine and are used without any further purification. Melting points were determined by the conventional method and then by electrocapillary apparatus and were uncorrected. All the synthesized compounds are inspected by thin layer chromatography (TLC) with precoated aluminium sheets on silica gel (E-Merck) and the spots are visualized by UV lamp. The IR spectra of the compounds are recorded on Shimadzu FT-IR spectrometer. ${ }^{1} \mathrm{H}$ NMR and ${ }^{13} \mathrm{C}$ NMR spectra are recorded using a Bruker in DMSO at $500 \mathrm{MHz}$. IR, ${ }^{1} \mathrm{H}$ NMR and ${ }^{13} \mathrm{C}$ NMR spectra were performed at centre of excellence saurashtra university and highresolution liquid chromatography-mass spectra were performed at the SAIF Indian institution of technology. Aminochalcone compounds 1a-1c are synthesized as shown in Scheme 1. Commercially available sulphonamides 2a-2e are treated with chloroacetyl chloride to provide chlorosulfonyl acetamide as shown in Scheme 2. The general route for the synthesis of the target sulphonamide-chalcone hybrids is depicted in Scheme 3. The structures of targeted compounds are characterized using spectral methods. All spectral data are fascinated with assumed structures.

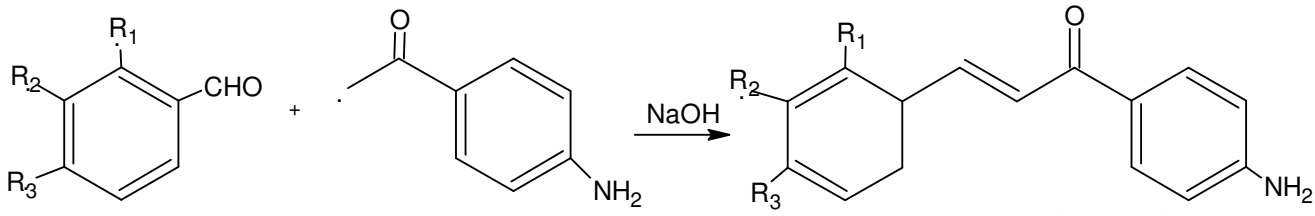

1a: $\mathrm{R}_{1}=\mathrm{H}_{1} \mathrm{R}_{2}=-\mathrm{OH}, \mathrm{R}_{3}=-\mathrm{CH}_{3}, 1 \mathrm{~b}: \mathrm{R}_{1}=-\mathrm{CH}_{3}, \mathrm{R}_{2}=-\mathrm{OH}, \mathrm{R}_{3}=-\mathrm{CH}_{3}, 1 \mathrm{c}: \mathrm{R}_{1}=-\mathrm{OH}, \mathrm{R}^{2}=-\mathrm{H}, \mathrm{R}^{3}=-\mathrm{H}$

Scheme 1. Synthesis of aminochalcone 
<smiles>[R]NS(=O)(=O)c1ccc(N)cc1</smiles>

$2 \mathbf{a}-2 \dot{e}$<smiles>CCN1CCCCC1</smiles>

Scheme 2. Synthesis of chlorosulfonyl acetamide<smiles>[R]NS(=O)(=O)c1ccc(NC(=O)CCl)cc1</smiles>

[II]<smiles>[R]NNS(=O)(=O)c1ccc(NC(=O)CNc2ccc(C(=O)/C=C/c3ccc([R3])c([R])c3[R])cc2)cc1</smiles>

Scheme 3. Synthesis of chalcone-sulphonamide hybrids

\section{Synthesis}

\section{General procedure of synthesis of aminochalcone(1a-1c)}

The synthesis of chalcone derivatives (I) was conducted according to the procedure reported in the reference by Claisen-Schmidt condensation reaction ${ }^{10-12}$. Acetophenone derivative $(2.5 \mathrm{mmol})$ and substituted benzaldehydes $(2.5$ milimole $)$ were dissolved in $30 \mathrm{~mL}$ methanol. To the solution, $10 \mathrm{~mL} \mathrm{NaOH}(20 \%)$ solution was added dropwise and the reaction mixture was stirred for 1-2 hours at room temperature by a magnetic stirrer and kept for overnight. Subsequently, it was poured into ice water and neutralized. The solid precipitates were filtered off and recrystallized from methanol or ethyl acetate (Table 1).

\section{General procedure of synthesis of intermediate(2a-2f)}

To a stirred solution of sulphonamide $(2 \mathrm{mmol})$ in dimethylformamide at $0-5{ }^{\circ} \mathrm{C}$, chloroacetyl chloride $(6 \mathrm{~mL})$ was added dropwise and stirred at room temperature for 3-4 hours by a magnetic stirrer. The reaction mixture was neutralized with triethylamine then the precipitate was filtered, washed with cold water recrystallized from methanol (Table 1).

\section{General procedure of synthesis of sulphonamide chalcone(3a-5f)}

To a solution of aminochalcone 1a-1c $(2 \mathrm{mmol})$ in dimethylformamide, a solution of an intermediate 2a-2f $(2 \mathrm{mmol})$ prepared in DMF was added. The stirred reaction mixture was then refluxed for 8-9 hours. Upon completion, the reaction mixture was poured to crushed ice. Precipitate was then washed with cold water and the crude product was recrystallized in acetone (Table 1).

Table 1. Structures and \% yield of chalcone-sulphonamide hybrids

\begin{tabular}{|c|c|c|c|c|}
\hline Entry & $\begin{array}{c}\text { Aldehyde } \\
\text { (1a-1c) }\end{array}$ & $\begin{array}{c}\text { Sulfonamide } \\
(\mathbf{2 a - 2 e})\end{array}$ & $\operatorname{Product}(\mathbf{3 a}-\mathbf{5 e})$ & $\begin{array}{c}\text { Yield, } \\
\%\end{array}$ \\
\hline 1 & 1a & & & $60 \%$ \\
\hline 2 & $1 \mathbf{a}$ & $2 \mathrm{~b}$ & & $50.5 \%$ \\
\hline 3 & $1 \mathbf{a}$ & $2 \mathrm{c}$ & 30 & $62.0 \%$ \\
\hline
\end{tabular}




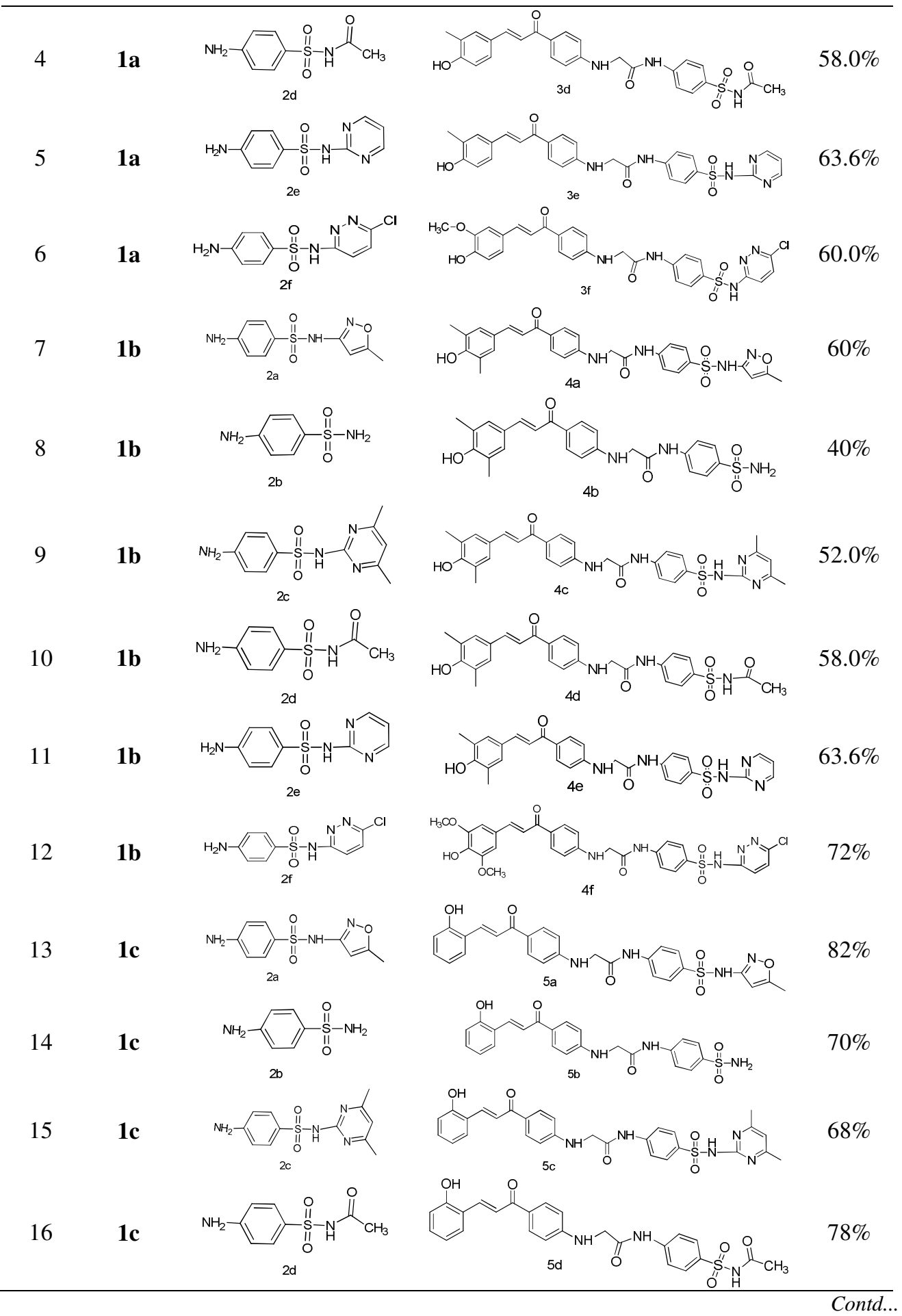




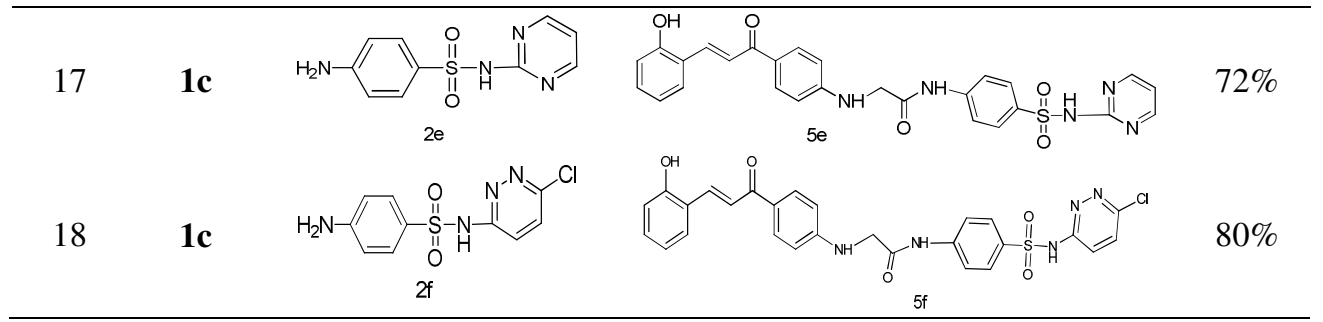

\section{Determination of antimicrobial activity}

Antibacterial activity of all the synthesized chalcone-sulphonamide hybrids was assayed against Pseudomonas aeruginosa and Staphylococcus epidermidis bacterial strains and antifungal activity was tested against Candida albicans fungi using antimicrobial susceptibility test approved by national committee for clinical laboratory standards (NCCLS). The minimum inhibitory concentration (MIC) of compounds for each test organism was assessed by a modification of the broth dilution method performed in 96 well micro-trays (NCCLS, 1993). A stock solution of the extract was made in dimethylsulphoxide (DMSO). Three-fold serial dilutions of the reconstituted compounds $(150 \mu \mathrm{L})$ of each sample were made in sterile broth (nutrient broth) to achieve a concentration range of $1.95-1000 \mu \mathrm{g} / \mathrm{mL}$. A number of test organisms $(50 \mu \mathrm{L})$ were added to each dilution to give a final volume of $200 \mu \mathrm{L}$. After incubation at $37{ }^{\circ} \mathrm{C}$ for $18-24 \mathrm{~h}$ the plates were examined for growth of the organisms. The lowest concentration that inhibited growth was recorded as the MIC. Growth was determined as the difference in absorbance at $595 \mathrm{~nm}$ between reading taken before and after the incubation of the plates. Readings less than $20 \%$ of the positive control were recorded as inhibition. Absorbance was read in a plate reader. Positive $(50 \mu \mathrm{L}$ cells $+150 \mu \mathrm{L}$ medium $)$ and negative $(200 \mu \mathrm{L}$ medium) controls were performed with each experiment. Each plate also included a solvent control in which $100 \mu \mathrm{L}$ of the appropriate diluted solvent was added to $100 \mu \mathrm{L}$ of the test organism. The data analysis was undertaken using Graph Pad InStat version 3.00 for Windows 95, Graph Pad Software Inc., San Diego California USA. IC50 values were obtained from regression lines with coefficient factors between $R^{2}=0.52$ and 0.99 . All the results of antimicrobial tests are listed in Table 2.

\section{Results and Discussion}

\section{Chemistry}

Commercially available para aminoacetophenone and substituted aldehydes are chosen to synthesize chalcone scaffold 1a-1c via base-catalyzed Claisen-Schmidt condensation ${ }^{22,23}$ using $10 \% \mathrm{NaOH}$ in methanol. All the synthesized chalcone derivatives are evaluated through IR and NMR spectral data. IR spectra of chalcone derivatives indicate the characteristic bands for carbonyl at $1650, \mathrm{CH}=\mathrm{CH}$ at $1590-1610$ and for $-\mathrm{OH}$ at 3570 $3395 \mathrm{~cm}^{-1}$. The ${ }^{1} \mathrm{H}$ NMR spectra show broad singlet at $\delta 3.47-3.50 \mathrm{ppm}$ appeared for $-\mathrm{NH}_{2}$ group, singlet of methoxy proton appeared about at $\delta 3.87 \mathrm{ppm}$ and multiplets at $\delta 7.20-7.90$ for phenyl protons. For $\mathrm{CO}-\mathrm{CH}=\mathrm{CH}$ one doublet appears at $\delta 7.51-7.68 \mathrm{ppm}$ and another doublet at $\delta$ 6.02-6.57 ppm respectively. The chlorosulphonamide derivatives $\mathbf{2 a - 2 f}$ have been synthesized by treatment of chloroacetyl chloride with a variety of sulpha drugs containing amino group in their structure in the presence of triethylamine $\left(\mathrm{Et}_{3} \mathrm{~N}\right)$.

Chalcone 1a-1c then has been condensed with chlorosulphonamide derivatives $2 \mathbf{a - 2 f}$ in dimethylformamide with a few drops of $\mathrm{Et}_{3} \mathrm{~N}$ resulted in chalcone-sulphonamide hybrids 3a-5f (c.f. Scheme 3). The noteworthy reaction observed is the formation of a C-N bond 
between the nitrogen of chalcone and carbon of sulphonamide derivatives. Structures of all the obtained hybrids 3a-5f are further supported by IR, NMR and HRMS. IR spectra of 3a5f display - NH- absorption band at $3330-3360 \mathrm{~cm}^{-1}$ and stretching band of amide carbonyl at 1734-1739 $\mathrm{cm}^{-1}$. The broad singlet in ${ }^{1} \mathrm{H}$ NMR of 3a-5f has disappeared which implies the absence of free $-\mathrm{NH}_{2}$ group of chalcone moiety and further resulted in the formation of a $\mathrm{C}-\mathrm{N}$ bond between two pharmacophores. In the ${ }^{1} \mathrm{H}$ NMR spectra, methylene protons present between -NH- and -CO- appear as a doublet at $\delta 3.36 \mathrm{ppm} .{ }^{13} \mathrm{C}$ NMR spectrum of 3a revealed different characteristic signals at $\delta 44.54$ for methylene, 56.79 for methoxy, 167.97 for amide carbonyl and 190.97 for vinyl carbonyl also reinforce the proposed structure. High-resolution mass spectroscopy reveals that hybrid 3a showed molecular ion $\left(\mathrm{M}+\mathrm{H}^{+}\right)$ peak at 546.157 corresponding to the molecular formula of $\mathrm{C}_{28} \mathrm{H}_{26} \mathrm{~N}_{4} \mathrm{O}_{6} \mathrm{~S}$.

Table 2. Percentage cell inhibition by compounds against S.epidermidis (gram-positive bacteria), P.aeruginosa (gram-negative bacteria) and Candida albicans fungi strain

\begin{tabular}{|c|c|c|c|c|c|c|}
\hline \multirow{2}{*}{ Entry } & \multicolumn{2}{|c|}{$\begin{array}{c}\text { Staphylococcus } \\
\text { epidermidis }\end{array}$} & \multicolumn{2}{|c|}{$\begin{array}{c}\text { Pseudomonas } \\
\text { aeruginosa }\end{array}$} & \multicolumn{2}{|c|}{ Candida albicans } \\
\hline & $\begin{array}{c}\mathrm{IC}_{50} \\
\mathrm{uM} / \mathrm{mL}\end{array}$ & $\begin{array}{c}\text { Selectivity } \\
\text { Ratio }\end{array}$ & $\begin{array}{c}\mathrm{IC}_{50} \\
\mathrm{uM} / \mathrm{mL}\end{array}$ & $\begin{array}{c}\text { Selectivity } \\
\text { Ratio }\end{array}$ & $\begin{array}{c}\mathrm{IC}_{50} \\
\mathrm{uM} / \mathrm{mL}\end{array}$ & $\begin{array}{c}\text { Selectivity } \\
\text { Ratio }\end{array}$ \\
\hline $3 a$ & $>100$ & $<0.1$ & 41.73 & 0.149 & 21.30 & 0.484 \\
\hline $3 b$ & 20.24 & 0.426 & 20.24 & 0.308 & $>100$ & $<0.1$ \\
\hline $3 c$ & 32.40 & 0.266 & 22.95 & 0.272 & 100 & 0.10 \\
\hline $3 d$ & 20.71 & 0.416 & 14.52 & 0.432 & 16.34 & 0.630 \\
\hline $3 e$ & 42.22 & 0.204 & 5.94 & 1.050 & $>100$ & $<0.1$ \\
\hline $3 f$ & $>100$ & $<0.1$ & 11.50 & 0.542 & 16.69 & 0.617 \\
\hline $4 a$ & 41.94 & 0.206 & 15.94 & 0.392 & $>100$ & $<0.1$ \\
\hline $4 b$ & 22.89 & 0.377 & 89.32 & $<0.1$ & 13.07 & 0.788 \\
\hline $4 c$ & 14.01 & 0.616 & 2.523 & 2.470 & $>100$ & $<0.1$ \\
\hline $4 d$ & 7.58 & 1.150 & $>100$ & $<0.1$ & 25.73 & 0.400 \\
\hline $4 \mathrm{e}$ & 16.19 & 0.533 & $>100$ & $<0.1$ & $>100$ & $<0.1$ \\
\hline $4 \mathrm{f}$ & 2.568 & 3.369 & 40.30 & 0.155 & 25.93 & 0.397 \\
\hline $5 a$ & 21.88 & 0.394 & 75.19 & $<0.1$ & 9.32 & 1.110 \\
\hline $5 b$ & $>100$ & $<0.1$ & 18.71 & 0.333 & 75.36 & 0.139 \\
\hline $5 c$ & 69.72 & 0.124 & 6.05 & 1.040 & 27.11 & 0.380 \\
\hline $5 d$ & 67.08 & 0.128 & $>100$ & $<0.1$ & 76.88 & 0.134 \\
\hline $5 e$ & 7.44 & 1.160 & $>100$ & $<0.1$ & $>100$ & $<0.1$ \\
\hline $5 f$ & 26.62 & 0.324 & 11.18 & 0.558 & 38.09 & 0.270 \\
\hline *STD & 8.625 & 1 & 6.233 & 1 & 10.30 & 1 \\
\hline
\end{tabular}

$* S T D=$ Streptomycin for bacterial strains and fluconazole for fungal strain

1a: (2E)-1-(4-Aminophenyl)-3-(4-hydroxy-3-methoxyphenyl) prop-2-en-1-one

Yellow solid, Yield 58.9\%. M.P 105-107 ${ }^{\circ} \mathrm{C}, \mathrm{R}_{\mathrm{f}} 0.71$ FT-IR $\left(v, \mathrm{~cm}^{-1}\right)$ : 3395(-OH), 3330, 3225(-NH $)_{2}$, 3063(aromatic C-H), $1649(>\mathrm{C}=\mathrm{O}), 1590(-\mathrm{HC}=\mathrm{CH}-), 1277,1305(\mathrm{C}-\mathrm{N}$ str) ${ }^{1} \mathrm{H}$ NMR (500 MHz DMSO, $\left.\mathrm{Me}_{4} \mathrm{Si}\right): 3.50\left(\mathrm{br}, \mathrm{s},-\mathrm{NH}_{2}\right), 3.85\left(\mathrm{~s},-\mathrm{OCH}_{3}\right), 6.027\left(\mathrm{~s}, 1 \mathrm{H}, \mathrm{H}_{2}\right)$, 6.62( d, $\left.1 \mathrm{H}_{\alpha}\right), 7.66\left(\mathrm{~d}, 1 \mathrm{H}_{\beta}\right)$. GC-MS (EI, $\left.\mathrm{m} / z\right): 269\left(\mathrm{M}^{+}\right), 266,239,214,191,171,99,62$.

lb: (2E)-1-(4-Aminophenyl)-3-(4-hydroxy-3,5-dimethoxyphenyl)prop-2-en-1-one

Yellow solid, Yield 57.5\%. M.P 98-100 ${ }^{\circ} \mathrm{C}, \mathrm{R}_{\mathrm{f}} 0.66$ FT-IR $\left(v, \mathrm{~cm}^{-1}\right)$ : 3396(-OH), 3331, 3225(- $\left.\mathrm{NH}_{2}\right), 3064$ (aromatic C-H), 1648 (>C=O), 1590 (-HC=CH-), 1276, 1304 (C-N str) 
${ }^{1} \mathrm{H}$ NMR (500 MHz DMSO, $\left.\mathrm{Me}_{4} \mathrm{Si}\right): 3.49\left(\mathrm{br}, \mathrm{s},-\mathrm{NH}_{2}\right), 3.71\left(\mathrm{~s},-\mathrm{OCH}_{3}\right), 3.82\left(\mathrm{~s},-\mathrm{OCH}_{3}\right)$, 6.028(s,1H, $\left.\mathrm{H}_{1}, \mathrm{H}_{6}\right), 6.58\left(\mathrm{~d}, 1 \mathrm{H}_{\alpha}\right), 7.68\left(\mathrm{~d}, 1 \mathrm{H}_{\beta}\right)$. GC-MS (EI, m/z): 299( $\left.\mathrm{M}^{+}\right), 266,229,214$, $181,166,62$.

1c: (2E)-1-(4-Aminophenyl)-3-(2-hydroxyphenyl)prop-2-en-1-one

Pale Yellow solid, Yield 62.5\%. M.P $110-112{ }^{\circ} \mathrm{C}, \mathrm{R}_{\mathrm{f}} 0.68$ FT-IR $\left(v, \mathrm{~cm}^{-1}\right): 3570(-\mathrm{OH})$, 3338, $3328\left(-\mathrm{NH}_{2}\right), 3050$ (aromatic $\left.\mathrm{C}-\mathrm{H}\right), 1676(>\mathrm{C}=\mathrm{O}), 1595(-\mathrm{HC}=\mathrm{CH}-), 1270,1364(\mathrm{C}-\mathrm{N}$ str) ${ }^{1} \mathrm{H}$ NMR (500 MHz DMSO, $\mathrm{Me}_{4} \mathrm{Si}$ ): 3.47 (br, s, $\left.-\mathrm{NH}_{2}\right), 6.57\left(\mathrm{~d}, 1 \mathrm{H}_{\alpha}\right), 7.51\left(\mathrm{~d}, 1 \mathrm{H}_{\beta}\right)$, $7.013\left(\mathrm{~m}, \mathrm{H}_{2}\right), 7.72\left(\mathrm{~m}, \mathrm{H}_{3}, \mathrm{H}_{4}\right)$. GC-MS (EI, $\left.\mathrm{m} / z\right)$ : 239(M $\left.{ }^{+}\right), 214,166,122,62$.

3a:(E)-2-((4-(3-(4-Hydroxy-3-methylphenyl)acryloyl)phenyl)amino)- $\mathrm{N}-(4-(\mathrm{N}-(5-$ methylisoxazol-3-yl) sulfamoyl)phenyl)acetamide

Yellow solid, $\mathrm{R}_{\mathrm{f}}$ 0.52. FT-IR ( $\left.v, \mathrm{~cm}^{-1}\right)$ : 3741(-OH), 3591, 3360,3331(3-NH-), 30643100(Ar C-H), 2974 (Aliphatic -CH), 1739 (-CONH-), 1678 (-CO), 1608 (-C=N), 1591 ($\mathrm{HC}=\mathrm{CH}-), 1454(\mathrm{C}-\mathrm{O}), 1398,1157\left(-\mathrm{SO}_{2^{-}}\right) 952(\mathrm{~S}-\mathrm{N}), 833(\mathrm{C}-\mathrm{S}) .{ }^{1} \mathrm{H}$ NMR $(500 \mathrm{MHz}$, DMSO-d $\left.\mathrm{d}_{6}, \quad \mathrm{ppm}\right): \quad 8.20 \quad(\mathrm{~s}, 1 \mathrm{H}, \quad \mathrm{CONH}-), \quad 8.10(\mathrm{~s}, 1 \mathrm{H},-\mathrm{OH}), \quad 7.65\left(\mathrm{~d}, 1 \mathrm{H}_{\beta} \quad-\mathrm{CH}=\mathrm{CH}-\right)$, 7.56(s,1H,- $\left.\mathrm{SO}_{2} \mathrm{NH}-\right), 6.45\left(\mathrm{~d}, 1 \mathrm{H}_{\alpha}-\mathrm{CH}=\mathrm{CH}-\right), 6.02-8.02(\mathrm{~m}, 10 \mathrm{H}, \mathrm{Ar}-\mathrm{H}), 6.51(\mathrm{t}, 1 \mathrm{H},-\mathrm{NH}-)$, $4.63(\mathrm{~s}, 1 \mathrm{H}, \quad \mathrm{CH}=\mathrm{C} \quad$ isoxazole $), \quad 3.36 \quad\left(\mathrm{~d}, 2 \mathrm{H},-\mathrm{CH}_{2}\right), \quad 2.67 \quad\left(\mathrm{~s}, 3 \mathrm{H},-\mathrm{CH}_{3}\right), \quad 2.27(\mathrm{~s}, 3 \mathrm{H},-$ $\left.\mathrm{OCH}_{3}\right) .{ }^{13} \mathrm{CNMR}\left(500 \mathrm{MHz}, \mathrm{DMSO}-\mathrm{d}_{6}, \mathrm{ppm}\right): 195.17,167.08,159.56,154.09,151.03$, 141.66, 144.34, 138.80, 130.80, 127.02, 126.00, 125.54, 122.95, 119.07, 119.07,112.40, 40.80, 39.67, 39.46,39.07, 38.83, 27.03, 25.92, 12.11. HR-MS (ESI) Calcd for $\mathrm{C}_{28} \mathrm{H}_{26} \mathrm{~N}_{4} \mathrm{O}_{6} \mathrm{~S}\left[\mathrm{M}+\mathrm{H}^{+}\right]$546.16, found 546.157.

3b:(E)-2-((4-(3-(4-Hydroxy-3-methylphenyl)acryloyl)phenyl)amino)-N-(4-sulfamoyl phenyl)acetamide

Brown solid, $\mathrm{R}_{\mathrm{f}}$ 0.56. FT-IR $\left(v, \mathrm{~cm}^{-1}\right)$ : 3741(-OH), 3591, 3566, 3365,3280(2-NH, $\left.-\mathrm{NH}_{2}\right)$, 3005(Ar C-H), 2976 (Aliphatic-CH), 1734 (-CONH-), 1683 (-CO), 1639 (-C=N), 1595 $(-\mathrm{HC}=\mathrm{CH}-), 1452(\mathrm{C}-\mathrm{O}), 1311,1149\left(-\mathrm{SO}_{2^{-}}\right), 962(\mathrm{~S}-\mathrm{N}), 829(\mathrm{C}-\mathrm{S}) .{ }^{1} \mathrm{H}$ NMR $(500 \mathrm{MHz}$,

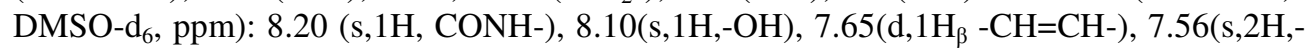
$\left.\mathrm{SO}_{2} \mathrm{NH}_{2}\right), 6.45\left(\mathrm{~d}, 1 \mathrm{H}_{\alpha}-\mathrm{CH}=\mathrm{CH}-\right), 6.02-8.02(\mathrm{~m}, 10 \mathrm{H}, \mathrm{Ar}-\mathrm{H}), 6.51(\mathrm{t}, 1 \mathrm{H},-\mathrm{NH}-), 3.13(\mathrm{~s}, 2 \mathrm{H},-$ $\left.\mathrm{CH}_{2}-\right), 2.67$ (s,3H,-OCH 3$), 2.40\left(\mathrm{~s}, 3 \mathrm{H},-\mathrm{CH}_{3}\right) .{ }^{13} \mathrm{CNMR}\left(500 \mathrm{MHz}, \mathrm{DMSO}-\mathrm{d}_{6}, \mathrm{ppm}\right): 195.17$, $169.08,159.56,154.08,141.66,138.80,130.54,129.62,127.02,126.70,125.54,122.95$, 117.17, 46.32, 40.09, 39.83,39.09, 38.83, 27.03, 25.92. HR-MS (ESI) Calcd for $\mathrm{C}_{24} \mathrm{H}_{23} \mathrm{~N}_{3} \mathrm{O}_{5} \mathrm{~S}$ $\left[\mathrm{M}+\mathrm{H}^{+}\right]$465.135, found 465.136.

3c:(E)-N-(4-(N-(4,6-Dimethylpyrimidin-2-yl)sulfamoyl)phenyl)-2-((4-(3-(4hydroxy-3-methylphenyl)acryloyl)phenyl)amino)acetamide

Brown solid, $\mathrm{R}_{\mathrm{f}}$ 0.52. FT-IR ( $\left.v \mathrm{~cm}^{-1}\right)$ : 3741(-OH), 3591, 3360,3253(3-NH-), 3000-3100(Ar C-H), 2974, 2883 (Aliphatic -CH), 1739 (-CONH-), 1678 (-CO), 1647 (-C=N ), 1593 $(-\mathrm{HC}=\mathrm{CH}-), 1456(\mathrm{C}-\mathrm{O}), 1396,1157\left(-\mathrm{SO}_{2^{-}}\right) 952(\mathrm{~S}-\mathrm{N}), 835(\mathrm{C}-\mathrm{S}) .{ }^{1} \mathrm{H}$ NMR $(500 \mathrm{MHz}$, DMSO-d $\left.\mathrm{d}_{6}, \mathrm{ppm}\right): 8.31$ (s,1H, CONH- $), 8.30(\mathrm{~s}, 1 \mathrm{H},-\mathrm{OH}), 7.65\left(\mathrm{~d}, 1 \mathrm{H}_{\beta}-\mathrm{CH}=\mathrm{CH}-\right), 7.56(\mathrm{~s}, 1 \mathrm{H},-$ $\left.\mathrm{SO}_{2} \mathrm{NH}-\right), 6.55\left(\mathrm{~d}, 1 \mathrm{H}_{\alpha}-\mathrm{CH}=\mathrm{CH}-\right), 6.09-8.02(\mathrm{~m}, 10 \mathrm{H}, \mathrm{Ar}-\mathrm{H}), 6.45\left(1 \mathrm{H}, \mathrm{s},-\mathrm{CH}=\mathrm{CH}_{\text {(pyrimidine) }}\right)$, $6.41(\mathrm{t}, 1 \mathrm{H},-\mathrm{NH}-), 3.36\left(\mathrm{~d}, 2 \mathrm{H},-\mathrm{CH}_{2}-\right), 2.56\left(\mathrm{~s}, 3 \mathrm{H},-\mathrm{CH}_{3}\right), 2.54\left(\mathrm{~s}, 3 \mathrm{H},-\mathrm{OCH}_{3}\right), 2.31(\mathrm{~s}, 3 \mathrm{H},-$ $\left.\mathrm{CH}_{3}\right){ }^{13} \mathrm{CNMR}$ (500 MHz, DMSO-d 6 , ppm): 195.17, 167.08, 159.56, 154.09,154.09 141.66, $138.80,130.80,129.24,127.02,127.02,126.00,125.54,122.95,112.40,119.02,119.12$, 114.40, 114.40, 40.80, 39.67, 39.46,39.07, 38.83, 27.03, 12.11. HR-MS (ESI) Calcd for $\mathrm{C}_{30} \mathrm{H}_{29} \mathrm{~N}_{5} \mathrm{O}_{5} \mathrm{~S}\left[\mathrm{M}+\mathrm{H}^{+}\right]$571.18, found 571.211. 
3d:(E)-N-(4-(N-Acetylsulfamoyl)phenyl)-2-((4-(3-(4-hydroxy-3-methylphenyl)acryloyl) phenyl)amino)acetamide

Red solid, $\mathrm{R}_{\mathrm{f}}$ 0.70. FT-IR ( $\left.v, \mathrm{~cm}^{-1}\right)$ : 3741(-OH), 3568, 3360,3246 (3-NH-), 3000(Ar C-H), 2976,2889 (Aliphatic -CH ), 1734 (-CONH-), 1678 (-CO), 1647 (-C=N ), 1597 (-HC=CH-), 1456(C-O), 1363, 1155 (-SO $\left.2^{-}\right), 958$ (S-N), 839(C-S). ${ }^{1} \mathrm{H}$ NMR (500 MHz, DMSO-d 6 , ppm): $8.20(\mathrm{~s}, 1 \mathrm{H}, \quad \mathrm{CONH}-), \quad 8.10(\mathrm{~s}, 1 \mathrm{H},-\mathrm{OH}), \quad 7.84\left(\mathrm{~d}, 1 \mathrm{H}_{\beta} \quad-\mathrm{CH}=\mathrm{CH}-\right), \quad 7.76\left(\mathrm{~s}, 1 \mathrm{H},-\mathrm{SO}_{2} \mathrm{NH}-\right)$, $6.45\left(\mathrm{~d}, 1 \mathrm{H}_{\alpha}-\mathrm{CH}=\mathrm{CH}-\right), 6.02-8.02(\mathrm{~m}, 10 \mathrm{H}, \mathrm{Ar}-\mathrm{H}), 6.51(\mathrm{t}, 1 \mathrm{H},-\mathrm{NH}-), 3.35\left(\mathrm{~d}, 2 \mathrm{H},-\mathrm{CH}_{2}-\right), 2.67$ $\left(\mathrm{s}, 3 \mathrm{H},-\mathrm{CH}_{3}\right), \quad 2.40\left(\mathrm{~s}, 3 \mathrm{H},-\mathrm{OCH}_{3}\right) .{ }^{13} \mathrm{CNMR}$ (500 MHz, DMSO-d $\left.\mathrm{d}_{6}, \mathrm{ppm}\right):$ 190.17,170.20, $167.08,159.56$, 154.09, 141.66, 138.80, 130.80, 129.06, 129.06, 127.02, 122.95, 119.08, 119.12, 114.40, 44.80, 39.67, 39.46, 39.07, 38.83, 21.03, 16.11. HR-MS (ESI) Calcd for $\mathrm{C}_{26} \mathrm{H}_{25} \mathrm{~N}_{3} \mathrm{O}_{6} \mathrm{~S}\left[\mathrm{M}+\mathrm{H}^{+}\right]$507.14, found 507.23.

3e:(E)-2-((4-(3-(4-Hydroxy-3-methylphenyl)acryloyl)phenyl)amino)- $N$-(4-(N(pyrimidin-2-yl) sulfamoyl) phenyl)acetamide

Red brick solid, $\mathrm{R}_{\mathrm{f}}$ 0.41 FT-IR ( $\left.v, \mathrm{~cm}^{-1}\right)$ : 3743(-OH), 3589, 3360,3153(3-NH-), 3000-3100(Ar C-H), 2972, 2918 (Aliphatic -CH), 1738 (-CONH-), 1674 (-CO), 1649 (-C=N ), 1590 $(-\mathrm{HC}=\mathrm{CH}-), 1463(\mathrm{C}-\mathrm{O}), 1399,1155\left(-\mathrm{SO}_{2}-\right), 954(\mathrm{~S}-\mathrm{N}), 835(\mathrm{C}-\mathrm{S}) .{ }^{1} \mathrm{H}$ NMR (500 MHz,

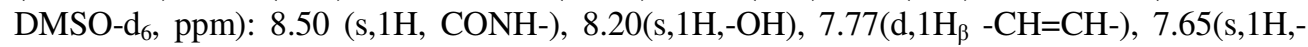
$\left.\mathrm{SO}_{2} \mathrm{NH}-\right), \quad 6.54\left(\mathrm{~d}, 1 \mathrm{H}_{\alpha}-\mathrm{CH}=\mathrm{CH}-\right), \quad 6.09-8.05(\mathrm{~m}, 10 \mathrm{H}, \mathrm{Ar}-\mathrm{H}), \quad 6.65\left(1 \mathrm{H}, \mathrm{s},-\mathrm{CH}=\mathrm{CH}_{\text {(pyrimidine) }}\right)$, 6.41(t, $1 \mathrm{H},-\mathrm{NH}-), 3.35\left(\mathrm{~d}, 2 \mathrm{H},-\mathrm{CH}_{2}-\right), 2.38\left(\mathrm{~s}, 3 \mathrm{H},-\mathrm{OCH}_{3}\right) .{ }^{13} \mathrm{CNMR}\left(500 \mathrm{MHz}, \mathrm{DMSO}-\mathrm{d}_{6}, \mathrm{ppm}\right)$ : $194.87,167.08,159.56,154.09,153.58,138.80,130.80,129.24,127.02,124.78,122.95$, 112.40, 119.02,119.12,114.40, 114.40, 40.80, 39.67, 39.46,39.25, 39.07, 38.83, 27.03, 25.11 . HR-MS (ESI) Calcd for $\mathrm{C}_{28} \mathrm{H}_{25} \mathrm{~N}_{5} \mathrm{O}_{5} \mathrm{~S}\left[\mathrm{M}+\mathrm{H}^{+}\right]$543.157, found 543.159.

3f:(E)-N-(4-(N-(6-Chloropyridazin-3-yl)sulfamoyl)phenyl)-2-((4-(3-(4-hydroxy-3methoxyphenyl) acryloyl)phenyl)amino) acetamide

Black solid, $\mathrm{R}_{\mathrm{f}}$ 0.61 FT-IR $\left(v, \mathrm{~cm}^{-1}\right): 3750(-\mathrm{OH}), 3585,3356,3143(3-\mathrm{NH}-)$, 3000-3100(Ar CH), 2972, 2918 (Aliphatic -CH), $1738(-\mathrm{CONH}-), 1674(-\mathrm{CO}), 1649(-\mathrm{C}=\mathrm{N}), 1590(-\mathrm{HC}=\mathrm{CH}-)$, 1463(C-O), 1399, $1155\left(-\mathrm{SO}_{2}-\right), 954(\mathrm{~S}-\mathrm{N}), 835(\mathrm{C}-\mathrm{S}), 595(\mathrm{C}-\mathrm{Cl}) .{ }^{1} \mathrm{H}$ NMR $(500 \mathrm{MHz}$, DMSO-d $\left.\mathrm{d}_{6}, \mathrm{ppm}\right): 8.50$ (s,1H, CONH-), $8.20(\mathrm{~s}, 1 \mathrm{H},-\mathrm{OH}), 7.77\left(\mathrm{~d}, 1 \mathrm{H}_{\beta}-\mathrm{CH}=\mathrm{CH}-\right), 7.65(\mathrm{~s}, 1 \mathrm{H},-$ $\left.\mathrm{SO}_{2} \mathrm{NH}-\right), 6.54\left(\mathrm{~d}, 1 \mathrm{H}_{\alpha}-\mathrm{CH}=\mathrm{CH}-\right), 6.09-8.05(\mathrm{~m}, 10 \mathrm{H}, \mathrm{Ar}-\mathrm{H}), 6.65\left(1 \mathrm{H}, \mathrm{s},-\mathrm{CH}=\mathrm{CH}_{\text {(pyrimidine) }}\right)$, 6.41(t, $1 \mathrm{H},-\mathrm{NH}-), 3.35\left(\mathrm{~d}, 2 \mathrm{H},-\mathrm{CH}_{2}-\right), 2.38\left(\mathrm{~s}, 3 \mathrm{H},-\mathrm{OCH}_{3}\right) .{ }^{13} \mathrm{CNMR}\left(500 \mathrm{MHz}, \mathrm{DMSO}-\mathrm{d}_{6}\right.$, ppm): 194.87, 167.08, 159.56, 154.09, 153.58, 146.52, 138.80, 130.80, 129.24, 127.02, 124.78, $122.95,112.40,119.02,119.12,114.40,114.40,40.80,39.67,39.46,39.25,39.07,38.83$, 27.03, 25.11. HR-MS (ESI) Calcd for $\mathrm{C}_{28} \mathrm{H}_{24} \mathrm{ClN}_{5} \mathrm{O}_{6} \mathrm{~S}\left[\mathrm{M}+\mathrm{H}^{+}\right]$593.118, found 593.113.

4a:(E)-2-((4-(3-(4-Hydroxy-3,5-dimethylphenyl)acryloyl)phenyl)amino)- $N$-(4-( $N$ (5-methylisoxazol-3-yl) sulfamoyl)phenyl)acetamide

Red solid, $\mathrm{R}_{\mathrm{f}}$ 0.51. FT-IR ( $\left.v, \mathrm{~cm}^{-1}\right)$ : 3741(-OH), 3566, 3360,3331(3-NH-), 3064-3100(Ar C$\mathrm{H}), 2974,2883$ (Aliphatic -CH), 1772 (-CONH-), 1678 (-CO), $1653 \quad(-\mathrm{C}=\mathrm{N}), 1591$ $(-\mathrm{HC}=\mathrm{CH}-), 1456(\mathrm{C}-\mathrm{O}), 1398,1159\left(-\mathrm{SO}_{2^{-}}\right) 952(\mathrm{~S}-\mathrm{N}), 831(\mathrm{C}-\mathrm{S}) .{ }^{1} \mathrm{H}$ NMR $(500 \mathrm{MHz}$,

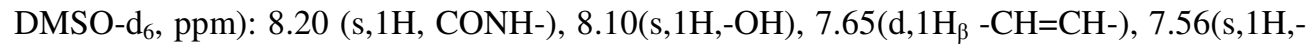
$\left.\mathrm{SO}_{2} \mathrm{NH}-\right), 6.45\left(\mathrm{~d}, 1 \mathrm{H}_{\alpha}-\mathrm{CH}=\mathrm{CH}-\right), 6.02-8.02(\mathrm{~m}, 10 \mathrm{H}, \mathrm{Ar}-\mathrm{H}), 6.51(\mathrm{t}, 1 \mathrm{H},-\mathrm{NH}-), 4.63(\mathrm{~s}, 1 \mathrm{H}$, $\left.\mathrm{CH}=\mathrm{C}_{\text {isoxazole }}\right), \quad 3.36\left(\mathrm{~d}, 2 \mathrm{H},-\mathrm{CH}_{2}-\right), 2.67 \quad\left(\mathrm{~s}, 3 \mathrm{H},-\mathrm{CH}_{3}\right), \quad 2.50\left(\mathrm{~s}, 3 \mathrm{H},-\mathrm{OCH}_{3}\right), \quad 2.27(\mathrm{~s}, 3 \mathrm{H},-$ $\left.\mathrm{OCH}_{3}\right) .{ }^{13} \mathrm{CNMR}\left(500 \mathrm{MHz}, \mathrm{DMSO}-\mathrm{d}_{6}, \mathrm{ppm}\right)$ : 195.17, 167.08, 159.56, 154.09, 151.03, $141.66,144.34,138.80,130.80,127.02,126.00,125.54,122.95,119.07,119.07,112.40$, 40.80, 39.67, 39.46,39.07, 38.83, 27.03, 25.92, 12.11,12.03. HR-MS (ESI) Calcd for $\mathrm{C}_{29} \mathrm{H}_{28} \mathrm{~N}_{4} \mathrm{O}_{6} \mathrm{~S}\left[\mathrm{M}+\mathrm{H}^{+}\right]$560.173, found 560.175. 
4b:(E)-2-((4-(3-(4-Hydroxy-3,5-dimethylphenyl)acryloyl)phenyl)amino)- $N$-(4sulfamoyl phenyl)acetamide

Pale yellow solid, $\mathrm{R}_{\mathrm{f}}$ 0.69. FT-IR $\left(v, \mathrm{~cm}^{-1}\right)$ : 3741(-OH), 3591, 3566, 3365,3280(2-NH, $\left.-\mathrm{NH}_{2}\right)$, 3005(Ar C-H), 2976 (Aliphatic -CH), 1734 (-CONH-), 1683 (-CO), 1639 (-C=N), 1595 ($\mathrm{HC}=\mathrm{CH}-), 1452(\mathrm{C}-\mathrm{O}), 1311,1149\left(-\mathrm{SO}_{2}-\right), 962(\mathrm{~S}-\mathrm{N}), 829(\mathrm{C}-\mathrm{S}) .{ }^{1} \mathrm{H}$ NMR $(500 \mathrm{MHz}, \mathrm{DMSO}-$ $\left.\mathrm{d}_{6}, \mathrm{ppm}\right): 8.20(\mathrm{~s}, 1 \mathrm{H}, \mathrm{CONH}-), 8.10(\mathrm{~s}, 1 \mathrm{H},-\mathrm{OH}), 7.65\left(\mathrm{~d}, 1 \mathrm{H}_{\beta}-\mathrm{CH}=\mathrm{CH}-\right), 7.56\left(\mathrm{~s}, 2 \mathrm{H},-\mathrm{SO}_{2} \mathrm{NH}_{2}\right)$, $6.45\left(\mathrm{~d}, 1 \mathrm{H}_{\alpha}-\mathrm{CH}=\mathrm{CH}-\right), 6.02-8.02(\mathrm{~m}, 10 \mathrm{H}, \mathrm{Ar}-\mathrm{H}), 6.51(\mathrm{t}, 1 \mathrm{H},-\mathrm{NH}-), 3.13\left(\mathrm{~s}, 2 \mathrm{H},-\mathrm{CH}_{2}-\right), 2.67$ $\left(\mathrm{s}, 3 \mathrm{H},-\mathrm{CH}_{3}\right), 2.50\left(\mathrm{~s}, 3 \mathrm{H},-\mathrm{OCH}_{3}\right), 2.40\left(\mathrm{~s}, 3 \mathrm{H},-\mathrm{OCH}_{3}\right) .{ }^{13} \mathrm{C} \mathrm{NMR}\left(500 \mathrm{MHz}, \mathrm{DMSO}-\mathrm{d}_{6}, \mathrm{ppm}\right)$ : $195.17,169.08,159.56,154.08,141.66,138.80,130.54,129.62,127.02,126.70,125.54$, 122.95, 117.17, 46.32, 40.09, 39.83, 39.09, 38.83, 27.03, 25.92, 12.54. HR-MS (ESI) Calcd for $\mathrm{C}_{25} \mathrm{H}_{25} \mathrm{~N}_{3} \mathrm{O}_{7} \mathrm{~S}\left[\mathrm{M}+\mathrm{H}^{+}\right]$479.52, found 479.53 .

4c:(E)-N-(4-(N-(4,6-Dimethylpyrimidin-2-yl)sulfamoyl)phenyl)-2-((4-(3-(4hydroxy-3,5-dimethyl phenyl)acryloyl)phenyl)amino)acetamide

Black solid, $\mathrm{R}_{\mathrm{f}}$ 0.57. FT-IR ( $\left.\mathrm{v}, \mathrm{cm}^{-1}\right)$ : 3741(-OH), 3591, 3560,3329(3-NH-), 3000(Ar C-H), 2974, 2883 (Aliphatic-CH ), 1739 ( -CONH-), 1678 (-CO), 1645 (-C=N ), 1593 (-HC=CH-), 1456(C-O), 1396, 1157 (-SO $\left.2^{-}\right) 952(\mathrm{~S}-\mathrm{N}), 835(\mathrm{C}-\mathrm{S}) .{ }^{1} \mathrm{H}$ NMR (500 MHz, DMSO-d 6 , ppm): $8.31(\mathrm{~s}, 1 \mathrm{H}, \quad \mathrm{CONH}-), \quad 8.30(\mathrm{~s}, 1 \mathrm{H},-\mathrm{OH}), \quad 7.65\left(\mathrm{~d}, 1 \mathrm{H}_{\beta} \quad-\mathrm{CH}=\mathrm{CH}-\right), \quad 7.56\left(\mathrm{~s}, 1 \mathrm{H},-\mathrm{SO}_{2} \mathrm{NH}-\right)$, $6.55\left(\mathrm{~d}, 1 \mathrm{H}_{\alpha}-\mathrm{CH}=\mathrm{CH}-\right), 6.09-8.02(\mathrm{~m}, 10 \mathrm{H}, \mathrm{Ar}-\mathrm{H}), 6.45\left(1 \mathrm{H}, \mathrm{s},-\mathrm{CH}=\mathrm{CH}_{\text {(pyrimidine) }}\right) 6.41(\mathrm{t}, 1 \mathrm{H}$, -NH-), $3.36\left(\mathrm{~d}, 2 \mathrm{H},-\mathrm{CH}_{2}-\right), 2.56\left(\mathrm{~s}, 3 \mathrm{H},-\mathrm{CH}_{3}\right), 2.54\left(\mathrm{~s}, 3 \mathrm{H},-\mathrm{OCH}_{3}\right), 2.31\left(\mathrm{~s}, 3 \mathrm{H},-\mathrm{OCH}_{3}\right) .{ }^{13} \mathrm{CNMR}$ (500 MHz, DMSO-d 6 , ppm): 195.17, 167.08, 159.56, 154.09,154.09 141.66, 138.80, 130.80, $129.24,127.02,127.02,126.00,125.54,122.95,112.40,119.02,119.12,114.40,114.40$, 40.80, 39.67, 39.46,39.07, 38.83, 27.03, 12.78,12.10 HR-MS (ESI) Calcd for $\mathrm{C}_{31} \mathrm{H}_{31} \mathrm{~N}_{5} \mathrm{O}_{7} \mathrm{~S}$ $\left[\mathrm{M}+\mathrm{H}^{+}\right]$585.20, found 585.201.

4d:(E)-N-(4-(N-Acetylsulfamoyl)phenyl)-2-((4-(3-(4-hydroxy-3,5-dimethylphenyl) acryloyl)phenyl)amino)acetamide

Brown solid, $\mathrm{R}_{\mathrm{f}}$ 0.80. FT-IR ( $\left.v, \mathrm{~cm}^{-1}\right)$ : 3741(-OH), 3591, 3360,3255 (3-NH-), 3000-3100(Ar C-H), 2974,2883 (Aliphatic -CH), 1734 (-CONH-), 1678 (-CO), 1653 (-C=N ), 1591 $(-\mathrm{HC}=\mathrm{CH}-), 1456(\mathrm{C}-\mathrm{O}), 1363,1153\left(-\mathrm{SO}_{2}-\right), 952(\mathrm{~S}-\mathrm{N}), 829(\mathrm{C}-\mathrm{S}) .{ }^{1} \mathrm{H}$ NMR $(500 \mathrm{MHz}$, DMSO-d $\left._{6}, \mathrm{ppm}\right): 8.20$ (s,1H, CONH- $), 8.10(\mathrm{~s}, 1 \mathrm{H},-\mathrm{OH}), 7.84\left(\mathrm{~d}, 1 \mathrm{H}_{\beta}-\mathrm{CH}=\mathrm{CH}-\right), 7.76(\mathrm{~s}, 1 \mathrm{H},-$ $\left.\mathrm{SO}_{2} \mathrm{NH}-\right), 6.45\left(\mathrm{~d}, 1 \mathrm{H}_{\alpha}-\mathrm{CH}=\mathrm{CH}-\right), 6.02-8.02(\mathrm{~m}, 10 \mathrm{H}, \mathrm{Ar}-\mathrm{H}), 6.51(\mathrm{t}, 1 \mathrm{H},-\mathrm{NH}-), 3.35(\mathrm{~d}, 2 \mathrm{H},-$ $\left.\mathrm{CH}_{2}-\right), 2.67\left(\mathrm{~s}, 3 \mathrm{H},-\mathrm{CH}_{3}\right), \quad 2.50\left(\mathrm{~s}, 3 \mathrm{H},-\mathrm{OCH}_{3}\right), \quad 2.40\left(\mathrm{~s}, 3 \mathrm{H},-\mathrm{OCH}_{3}\right) .{ }^{13} \mathrm{CNMR}(500 \mathrm{MHz}$, DMSO-d $\left.{ }_{6}, \mathrm{ppm}\right): 190.17,170.20,167.08,159.56,154.09,141.66,138.80,130.80,129.06$, 129.06, 127.02, 122.95,119.08,119.12, 114.40, 44.80, 39.67, 39.46,39.07, 38.83, 21.03, 16.11,12.23. HR-MS (ESI) Calcd for $\mathrm{C}_{27} \mathrm{H}_{27} \mathrm{~N}_{3} \mathrm{O}_{6} \mathrm{~S}\left[\mathrm{M}+\mathrm{H}^{+}\right]$521.16, found 521.25.

4e:(E)-2-((4-(3-(4-Hydroxy-3,5-dimethylphenyl)acryloyl)phenyl)amino)- $N$-(4-(N(pyrimidin-2-yl)sulfamoyl)phenyl)acetamide

Red solid, $\mathrm{R}_{\mathrm{f}}$ 0.61 FT-IR ( $\left.v, \mathrm{~cm}^{-1}\right)$ : 3743(-OH), 3649, 3564, 3358(3-NH-), 3000-3100(Ar C$\mathrm{H}), 2974$, (Aliphatic -CH ), 1738 ( -CONH-), 1674 (-CO), 1687 (-C=N ), 1587 (-HC=CH-), 1463(C-O), 1363, $1153\left(-\mathrm{SO}_{2^{-}}\right), 950(\mathrm{~S}-\mathrm{N}), 829(\mathrm{C}-\mathrm{S}) .{ }^{1} \mathrm{H}$ NMR $\left(500 \mathrm{MHz}, \mathrm{DMSO}-\mathrm{d}_{6}, \mathrm{ppm}\right)$ : $8.50(\mathrm{~s}, 1 \mathrm{H}, \quad \mathrm{CONH}-), \quad 8.20(\mathrm{~s}, 1 \mathrm{H},-\mathrm{OH}), \quad 7.77\left(\mathrm{~d}, 1 \mathrm{H}_{\beta} \quad-\mathrm{CH}=\mathrm{CH}-\right), \quad 7.65\left(\mathrm{~s}, 1 \mathrm{H},-\mathrm{SO}_{2} \mathrm{NH}-\right)$, 6.54(d, $\left.1 \mathrm{H}_{\alpha}-\mathrm{CH}=\mathrm{CH}-\right), 6.09-8.05(\mathrm{~m}, 10 \mathrm{H}, \mathrm{Ar}-\mathrm{H}), 6.65\left(1 \mathrm{H}, \mathrm{s},-\mathrm{CH}=\mathrm{CH}_{\text {(pyrimidine) }}\right), 6.41(\mathrm{t}, 1 \mathrm{H},-$ $\mathrm{NH}-), 3.35\left(\mathrm{~d}, 2 \mathrm{H},-\mathrm{CH}_{2}-\right), 2.50\left(\mathrm{~s}, 3 \mathrm{H},-\mathrm{CH}_{3}\right), 2.38\left(\mathrm{~s}, 3 \mathrm{H},-\mathrm{OCH}_{3}\right) .{ }^{13} \mathrm{CNMR}(500 \mathrm{MHz}$, DMSO$\left.\mathrm{d}_{6}, \mathrm{ppm}\right): 194.87,167.08,159.56,154.09,153.58,138.80,130.80,129.24,127.02,124.78$, $122.95,112.40,119.02$, 119.12, 114.40, 114.40, 40.80, 39.67, 39.46, 39.25, 39.07, 38.83, 27.03, 25.81,16.12. HR-MS (ESI) Calcd for $\mathrm{C}_{29} \mathrm{H}_{27} \mathrm{~N}_{5} \mathrm{O}_{5} \mathrm{~S}\left[\mathrm{M}+\mathrm{H}^{+}\right]$557.173, found 557.173. 
4f:(E)-N-(4-(N-(6-Chloropyridazin-3-yl)sulfamoyl)phenyl)-2-((4-(3-(4-hydroxy-3,5dimethoxyphenyl)acryloyl)phenyl)amino)acetamide

Brown solid, $\mathrm{R}_{\mathrm{f}} 0.65$ FT-IR $\left(v, \mathrm{~cm}^{-1}\right)$ : 3745(-OH), 3638, 3560,3355(3-NH-), 3000-3100(Ar C-H), 2974, (Aliphatic -CH ), 1738 (-CONH-), 1674 (-CO), 1687 (-C=N), 1587 (-HC=CH), 1463(C-O), 1363, $1153\left(-\mathrm{SO}_{2}-\right), 950(\mathrm{~S}-\mathrm{N}), 829(\mathrm{C}-\mathrm{S}), 590.22(\mathrm{C}-\mathrm{Cl}) .{ }^{1} \mathrm{H}$ NMR $(500 \mathrm{MHz}$,

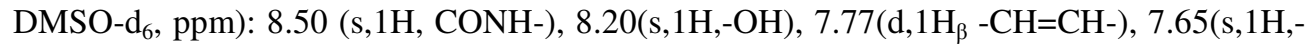
$\left.\mathrm{SO}_{2} \mathrm{NH}-\right), 6.54\left(\mathrm{~d}, 1 \mathrm{H}_{\alpha}-\mathrm{CH}=\mathrm{CH}-\right), 6.09-8.05(\mathrm{~m}, 10 \mathrm{H}, \mathrm{Ar}-\mathrm{H}), 6.65\left(1 \mathrm{H}, \mathrm{s},-\mathrm{CH}=\mathrm{CH}_{\text {(pyrimidine) }}\right)$, $6.41(\mathrm{t}, 1 \mathrm{H},-\mathrm{NH}-), 3.35\left(\mathrm{~d}, 2 \mathrm{H},-\mathrm{CH}_{2}-\right), 2.50\left(\mathrm{~s}, 3 \mathrm{H},-\mathrm{CH}_{3}\right), 2.38\left(\mathrm{~s}, 3 \mathrm{H},-\mathrm{OCH}_{3}\right) .{ }^{13} \mathrm{CNMR}(500$ MHz, DMSO-d $\left.\mathrm{d}_{6}, \mathrm{ppm}\right): 194.87,167.08,159.56,154.09,153.58,144.56,138.80,130.80$, $129.24,127.02,124.78,122.95,112.40,119.02,119.12,114.40,114.40,40.80,39.67$, $39.46,39.25,39.07,38.83,27.03,25.81,16.12$. HR-MS (ESI) Calcd for $\mathrm{C}_{29} \mathrm{H}_{26} \mathrm{ClN}_{5} \mathrm{O}_{7} \mathrm{~S}$ $\left[\mathrm{M}+\mathrm{H}^{+}\right]$623.140, found 623.124.

5a:(E)-2-((4-(3-(2-Hydroxyphenyl)acryloyl)phenyl)amino $)-\mathrm{N}-(4-(\mathrm{N}-(5-$ methylisoxazol-3-yl) sulfamoyl)phenyl)acetamide

Red solid, $\mathrm{R}_{\mathrm{f}}$ 0.68. FT-IR ( $\left.v, \mathrm{~cm}^{-1}\right)$ : 3741(-OH), 3672, 3568,3360,(3-NH-), 3064-3100(Ar C$\mathrm{H}), 2974,2883$ (Aliphatic-CH), 1734 (-CONH-), $1678(-\mathrm{CO}), 1608(-\mathrm{C}=\mathrm{N}), 1593(-\mathrm{HC}=\mathrm{CH}-)$, 1454(C-O), 1396, 1153 (-SO $\left.2^{-}\right) 954(\mathrm{~S}-\mathrm{N}), 831(\mathrm{C}-\mathrm{S}) .{ }^{1} \mathrm{H}$ NMR (500 MHz, DMSO-d 6 , ppm): $8.20(\mathrm{~s}, 1 \mathrm{H}, \quad \mathrm{CONH}-), \quad 8.10(\mathrm{~s}, 1 \mathrm{H},-\mathrm{OH}), \quad 7.65\left(\mathrm{~d}, 1 \mathrm{H}_{\beta} \quad-\mathrm{CH}=\mathrm{CH}-\right), \quad 7.56\left(\mathrm{~s}, 1 \mathrm{H},-\mathrm{SO}_{2} \mathrm{NH}-\right)$, 6.45(d,1 $\left.\mathrm{H}_{\alpha}-\mathrm{CH}=\mathrm{CH}-\right), 6.02-8.02(\mathrm{~m}, 10 \mathrm{H}, \mathrm{Ar}-\mathrm{H}), 6.51(\mathrm{t}, 1 \mathrm{H},-\mathrm{NH}-), 4.63(\mathrm{~s}, 1 \mathrm{H}, \mathrm{CH}=\mathrm{C}$ isoxazole $), 3.36\left(\mathrm{~d}, 2 \mathrm{H},-\mathrm{CH}_{2}-\right), 2.67\left(\mathrm{~s}, 3 \mathrm{H},-\mathrm{CH}_{3}\right) .{ }^{13} \mathrm{CNMR}$ (500 MHz, DMSO-d 6 , ppm): 195.17, $167.08,159.56,154.09,151.03,141.66,144.34,138.80,130.80,127.02,126.00,125.54$, $122.95,119.07,119.07,112.40,40.80,39.67,39.46,39.07,38.83,27.03,25.92$. HR-MS (ESI) Calcd for $\mathrm{C}_{27} \mathrm{H}_{24} \mathrm{~N}_{4} \mathrm{O}_{6} \mathrm{~S}\left[\mathrm{M}+\mathrm{H}^{+}\right]$532.14, found 532.14.

5b:(E)-2-((4-(3-(2-Hydroxyphenyl)acryloyl)phenyl)amino)-N-(4-sulfamoylphenyl) acetamide

Red solid, $\mathrm{R}_{\mathrm{f}}$ 0.56. FT-IR ( $\left.v, \mathrm{~cm}^{-1}\right)$ : 3741(-OH), 3591, 3566, 3365,3280(2-NH, $\left.-\mathrm{NH}_{2}\right)$, 3005(Ar C-H), 2976 (Aliphatic-CH), 1734 (-CONH-), 1683 (-CO), 1639 (-C=N), 1595 $(-\mathrm{HC}=\mathrm{CH}-), 1452(\mathrm{C}-\mathrm{O}), 1311,1149\left(-\mathrm{SO}_{2}-\right), 962(\mathrm{~S}-\mathrm{N}), 829(\mathrm{C}-\mathrm{S}) .{ }^{1} \mathrm{H}$ NMR $(500 \mathrm{MHz}$, DMSO-d $\left.\mathrm{d}_{6}, \mathrm{ppm}\right): 8.20$ (s,1H, CONH-), 8.10(s,1H,-OH), 7.65(d, $\left.1 \mathrm{H}_{\beta}-\mathrm{CH}=\mathrm{CH}-\right), 7.56(\mathrm{~s}, 2 \mathrm{H},-$ $\left.\mathrm{SO}_{2} \mathrm{NH}_{2}\right), 6.45\left(\mathrm{~d}, 1 \mathrm{H}_{\alpha}-\mathrm{CH}=\mathrm{CH}-\right), 6.02-8.02(\mathrm{~m}, 10 \mathrm{H}, \mathrm{Ar}-\mathrm{H}), 6.51(\mathrm{t}, 1 \mathrm{H},-\mathrm{NH}-), 3.13(\mathrm{~s}, 2 \mathrm{H},-$ $\left.\mathrm{CH}_{2}-\right) .{ }^{13} \mathrm{C}$ NMR $\left(500 \mathrm{MHz}, \mathrm{DMSO}-\mathrm{d}_{6}, \mathrm{ppm}\right): 195.17,169.08,159.56,154.08,141.66$, 138.80, 130.54, 129.62, 127.02, 126.70, 125.54, 122.95, 117.17, 46.32, 40.09, 39.83, 39.09, 38.83, 29.03. HR-MS (ESI) Calcd for $\mathrm{C}_{23} \mathrm{H}_{21} \mathrm{~N}_{3} \mathrm{O}_{5} \mathrm{~S}\left[\mathrm{M}+\mathrm{H}^{+}\right]$451.120, found 451.117.

5c:(E)-N-(4-(N-(4,6-Dimethylpyrimidin-2-yl)sulfamoyl)phenyl)-2-((4-(3-(2-hydroxy phenyl)acryloyl) phenyl)amino)acetamide

Red solid, $\mathrm{R}_{\mathrm{f}}$ 0.77. FT-IR ( $\left.v, \mathrm{~cm}^{-1}\right)$ : 3741(-OH), 3672,3591, 3360(3-NH-), 3000-3100(Ar C-H), 2974, 2883 (Aliphatic-CH), 1739 (-CONH-), 1678 (-CO), 1645 (-C=N), 1593 (-HC=CH-), 1454(C-O), 1396, 1155 (-SO $\left.2^{-}\right), 954(\mathrm{~S}-\mathrm{N}), 835(\mathrm{C}-\mathrm{S}) .{ }^{1} \mathrm{H}$ NMR $\left(500 \mathrm{MHz}, \mathrm{DMSO}-\mathrm{d}_{6}, \mathrm{ppm}\right)$ : $8.31(\mathrm{~s}, 1 \mathrm{H}, \quad \mathrm{CONH}-), \quad 8.30(\mathrm{~s}, 1 \mathrm{H},-\mathrm{OH}), \quad 7.65\left(\mathrm{~d}, 1 \mathrm{H}_{\beta} \quad-\mathrm{CH}=\mathrm{CH}-\right), \quad 7.56\left(\mathrm{~s}, 1 \mathrm{H},-\mathrm{SO}_{2} \mathrm{NH}-\right)$, $6.55\left(\mathrm{~d}, 1 \mathrm{H}_{\alpha}-\mathrm{CH}=\mathrm{CH}-\right), 6.09-8.02(\mathrm{~m}, 10 \mathrm{H}, \mathrm{Ar}-\mathrm{H}), 6.45\left(1 \mathrm{H}, \mathrm{s},-\mathrm{CH}=\mathrm{CH}_{\text {(pyrimidine) }}\right), 6.41(\mathrm{t}, 1 \mathrm{H},-$ $\mathrm{NH}-), 3.36\left(\mathrm{~d}, 2 \mathrm{H},-\mathrm{CH}_{2}-\right), 2.56\left(\mathrm{~s}, 3 \mathrm{H},-\mathrm{CH}_{3}\right), 2.31\left(\mathrm{~s}, 3 \mathrm{H},-\mathrm{CH}_{3}\right) .{ }^{13} \mathrm{CNMR}\left(500 \mathrm{MHz}, \mathrm{DMSO}-\mathrm{d}_{6}\right.$, ppm): 195.17, 167.08, 159.56, 154.09,154.09 141.66, 138.80, 130.80, 129.24, 127.02,127.02, $126.00,125.54,122.95,112.40,119.02,119.12,114.40,114.40,40.80,39.67,39.46,39.07$, 38.83, 27.03. HR-MS (ESI) Calcd for $\mathrm{C}_{29} \mathrm{H}_{27} \mathrm{~N}_{5} \mathrm{O}_{5} \mathrm{~S}\left[\mathrm{M}+\mathrm{H}^{+}\right]$557.62, found 557.62. 
5d:(E)-N-(4-(N-Acetylsulfamoyl)phenyl)-2-((4-(3-(2-hydroxyphenyl)acryloyl)phenyl) amino)acetamide

Red solid, $\mathrm{R}_{\mathrm{f}}$ 0.70. FT-IR $\left(v, \mathrm{~cm}^{-1}\right)$ : 3741(-OH), 3672,3568, 3360, (3-NH-), 3000-3100(Ar CH), 2972 (Aliphatic -CH ), 1739 ( -CONH-), 1678 (-CO), 1647 (-C=N), 1593 (-HC=CH-), 1456(C-O), 1396, 1155 (-SO $\left.2^{-}\right), 952$ (S-N), 835(C-S). ${ }^{1} \mathrm{H}$ NMR (500 MHz, DMSO-d 6 , ppm): $8.20(\mathrm{~s}, 1 \mathrm{H}, \quad \mathrm{CONH}-), \quad 8.10(\mathrm{~s}, 1 \mathrm{H},-\mathrm{OH}), \quad 7.84\left(\mathrm{~d}, 1 \mathrm{H}_{\beta} \quad-\mathrm{CH}=\mathrm{CH}-\right), \quad 7.76\left(\mathrm{~s}, 1 \mathrm{H},-\mathrm{SO}_{2} \mathrm{NH}-\right)$, 6.45(d,1 $\left.1 \mathrm{H}_{\alpha}-\mathrm{CH}=\mathrm{CH}-\right), 6.02-8.02(\mathrm{~m}, 10 \mathrm{H}, \mathrm{Ar}-\mathrm{H}), 6.51(\mathrm{t}, 1 \mathrm{H},-\mathrm{NH}-), 3.35\left(\mathrm{~d}, 2 \mathrm{H},-\mathrm{CH}_{2}-\right)$, 2.40(s,3H,-CH$).{ }^{13} \mathrm{C}$ NMR (500 MHz, DMSO-d 6 , ppm): 190.17, 170.20, 167.08, 159.56, $154.09,141.66,138.80,130.80,129.06,129.06,127.02$, 122.95,119.08,119.12, 114.40, 44.80, 39.67, 39.46,39.07, 38.83, 29.03. HR-MS (ESI) Calcd for $\mathrm{C}_{25} \mathrm{H}_{23} \mathrm{~N}_{3} \mathrm{O}_{6} \mathrm{~S}\left[\mathrm{M}+\mathrm{H}^{+}\right]$ 493.13, found 493.18.

5e:(E)-2-((4-(3-(2-Hydroxyphenyl)acryloyl)phenyl)amino)- $N-(4-(N-($ pyrimidin-2-yl) sulfamoyl)-phenyl)acetamide

Red Brick solid, $\mathrm{R}_{\mathrm{f}} 0.53$ FT-IR $\left(v, \mathrm{~cm}^{-1}\right)$ : 3743(-OH), 3672,3589, 3358(3-NH-), 30003100(Ar C-H), 2972 (Aliphatic-CH ), 1739 ( -CONH-), 1678 (-CO), 1649 (-C=N ), 1593 ($\mathrm{HC}=\mathrm{CH}-), 1456(\mathrm{C}-\mathrm{O}), 1369,1157\left(-\mathrm{SO}_{2}-\right), 952(\mathrm{~S}-\mathrm{N}), 833(\mathrm{C}-\mathrm{S}) .{ }^{1} \mathrm{H}$ NMR $(500 \mathrm{MHz}$, DMSO-d $\left.\mathrm{d}_{6}, \mathrm{ppm}\right): 8.50$ (s,1H, CONH-), 8.20(s,1H,-OH), 7.77(d, $\left.1 \mathrm{H}_{\beta}-\mathrm{CH}=\mathrm{CH}-\right), 7.65(\mathrm{~s}, 1 \mathrm{H},-$ $\left.\mathrm{SO}_{2} \mathrm{NH}-\right), 6.54\left(\mathrm{~d}, 1 \mathrm{H}_{\alpha}-\mathrm{CH}=\mathrm{CH}-\right), 6.09-8.05(\mathrm{~m}, 10 \mathrm{H}, \mathrm{Ar}-\mathrm{H}), 6.65\left(1 \mathrm{H}, \mathrm{s},-\mathrm{CH}=\mathrm{CH}_{\text {(pyrimidine) }}\right)$, $6.41(\mathrm{t}, \quad 1 \mathrm{H}, \quad-\mathrm{NH}-), \quad 3.41\left(\mathrm{~d}, 2 \mathrm{H},-\mathrm{CH}_{2}-\right) .{ }^{13} \mathrm{CNMR} \quad\left(500 \mathrm{MHz}, \quad \mathrm{DMSO}-\mathrm{d}_{6}, \quad \mathrm{ppm}\right)$ : $194.87,167.08,159.56,154.09, \quad 153.58,138.80, \quad 130.80, \quad 129.24,127.02,124.78, \quad 122.95$, $112.40,119.02,119.12,114.40,114.40,40.80,39.67,39.46,39.25,39.07,38.83,29.03$. HRMS (ESI) Calcd for $\mathrm{C}_{27} \mathrm{H}_{23} \mathrm{~N}_{5} \mathrm{O}_{5} \mathrm{~S}\left[\mathrm{M}+\mathrm{H}^{+}\right]$529.14, found 529.21.

5f:(E)-N-(4-(N-(6-Chloropyridazin-3-yl)sulfamoyl)phenyl)-2-((4-(3-(2-hydroxyphenyl) acryloyl)phenyl)amino)acetamide

Red Brick solid, $\mathrm{R}_{\mathrm{f}}$ 0.60 FT-IR ( $\left.v, \mathrm{~cm}^{-1}\right)$ : 3745(-OH), 3670,3578, 3358(3-NH-), 30003100(Ar C-H), 2972 (Aliphatic-CH ), 1739 ( -CONH-), 1678 (-CO), 1649 (-C=N ), 1593 ($\mathrm{HC}=\mathrm{CH}-), 1456(\mathrm{C}-\mathrm{O}), 1369,1157\left(-\mathrm{SO}_{2}-\right), 952(\mathrm{~S}-\mathrm{N}), 833(\mathrm{C}-\mathrm{S}), 597.93(\mathrm{C}-\mathrm{Cl}) .{ }^{1} \mathrm{H}$ NMR (500 MHz, DMSO-d 6 , ppm): $8.50(\mathrm{~s}, 1 \mathrm{H}, \mathrm{CONH}-), 8.20(\mathrm{~s}, 1 \mathrm{H},-\mathrm{OH}), 7.77\left(\mathrm{~d}, 1 \mathrm{H}_{\beta}-\mathrm{CH}=\mathrm{CH}-\right)$, 7.65(s, $\left.1 \mathrm{H},-\mathrm{SO}_{2} \mathrm{NH}-\right), \quad 6.54\left(\mathrm{~d}, 1 \mathrm{H}_{\alpha} \quad-\mathrm{CH}=\mathrm{CH}-\right), \quad 6.09-8.05(\mathrm{~m}, 10 \mathrm{H}, \quad \operatorname{Ar}-\mathrm{H}), \quad 6.65(1 \mathrm{H}, \mathrm{s},-$ $\left.\mathrm{CH}=\mathrm{CH}_{\text {(pyrimidine) }}\right), \quad 6.41(\mathrm{t}, \quad 1 \mathrm{H}, \quad-\mathrm{NH}-), \quad 3.41\left(\mathrm{~d}, 2 \mathrm{H},-\mathrm{CH}_{2^{-}}\right) .{ }^{13} \mathrm{CNMR} \quad$ (500MHz,DMSO$\left.\mathrm{d}_{6}, \mathrm{ppm}\right): 194.87,167.08,159.56,154.09,153.58,146.36,138.80,130.80,129.24,127.02$, 124.78, 122.95, 112.40, 119.02, 119.12, 114.40, 114.40, 40.80, 39.67, 39.46, 39.25, 39.07, 38.83, 29.03. HR-MS (ESI) Calcd for $\mathrm{C}_{27} \mathrm{H}_{22} \mathrm{ClN}_{5} \mathrm{O}_{5} \mathrm{~S}\left[\mathrm{M}+\mathrm{H}^{+}\right] 563.098$, found 563.103.

\section{Antimicrobial activity}

Chemically synthesized all chalcone-sulphonamide hybrids 3a-5f were screened for their antibacterial activity against P.aeruginosa (Gram-negative), S.epidermidis (Gram-positive) strains and also for their antifungal activity against Candida albicans. Table 2 lists the $\mathrm{IC}_{50}$ values and selectivity ratios against respective test microorganisms. Selectivity ratios of compounds are calculated using the following formula.

$$
\text { Selectivity ratio }=\frac{\mathrm{IC}_{50} \text { value of reference drug }}{\mathrm{IC}_{50} \text { value of synthesized compound }}
$$

The selectivity ratios of chalcone-sulphonamide hybrids are shown in Table 2. Arbitrarily, the $\mathrm{IC}_{50}$ value of synthesized hybrids against test organism is less than the $\mathrm{IC}_{50}$ values of reference drug and selectivity ratio $\geq 1$ are used to identify promising compounds. 
Results of gram-positive organism inhibition study indicate that $\mathbf{4 d}, \mathbf{4 f}$ and $5 \mathbf{e}$ hybrids are found to be dominant among others and their growth inhibitory potency towards S.epidermidis is in order $\mathbf{4 f}>\mathbf{5 e}>\mathbf{4 d}$. Similarly, results for gram-negative microorganism indicate that most effective compounds are arranged in the following order when it was compared to the $\mathrm{IC}_{50}$ value obtained by Streptomycin $(6.23 \mu \mathrm{M} / \mathrm{mL})$ with standard drug inhibitory value $4 \mathbf{c}>\mathbf{3 e}>\mathbf{5 c}$. Fluconazole and test compounds are tested against Candida albicans in a dose-dependent manner. Table 2 shows comparative lower $\mathrm{IC}_{50}$ value as found for 5a hybrid against Candida albicans.

\section{Conclusion}

Chalcone-sulphonamide hybrids have been fabricated by tethering sulphonamide pharmacophore with substituted chalcones with the aims of the multi-target drug for therapeutic treatment. Antibacterial and antifungal activities of the synthesized hybrids reveal that $\mathbf{4 d}$, $4 \mathbf{f}$ and $\mathbf{5 e}$ displayed a significant activity against Staphylococcus epidermidis and hybrids $\mathbf{3 e}, \mathbf{4 c}$ and $\mathbf{5 c}$ demonstrated a more potent growth inhibitory activity against Pseudomonas aeruginosa bacteria strains. Whereas 5a is more potent antifungal agent towards Candida albicans fungi. We may deduce that the sulphonamide pharmacophore possessing pyrimidine scaffold is associated with the enhanced antibacterial activity. This study may provide valuable research results for further investigation as therapeutic agents.

\section{Acknowledgement}

The authors Mahammadali Khanusiya and Zakirhusen Gadhawala gratefully acknowledge CSIR, New Delhi for MANF. The authors are also expressing their sincere thanks to The HNSB Ltd Science College for performing research work and SAIF IIT Mumbai for spectral analysis.

\section{Conflicts of Interest}

The authors reveal no conflict of interest.

\section{References}

1. Colliste C B, Bail L, Trouillas P, Pouget C, Habrioux G, Chulia A J and Duroux J L, Anticancer Res., 2001, 21(6A), 3949-3956.

2. Ngameni B, Kuete V, Ambassa P, Justine K, Marlyse M L, Med Chem., 2013, 3(3), 233-237; DOI:10.4172/2161-0444.1000144

3. Chandrabose K, Narayana S H, Narayana M, Sakhtivel R, Uma V, Elungavan M, Devrajan K and Piyush T, Recent Patents on Anticancer Drug Dis., 2015, 10, 97-115; DOI:10.2174/1574892809666140819153902

4. Chimenti F, Fioravanti R, Blasco A, Chimenti P, Secci D, Rossi F, Matilde Y, Francisco O, Francesco O and Stefano A, J Med Chem., 2009, 52(9), 2818-2824; DOI:10.1021/jm801590u

5. Kamal A, Prabhakar S, Ramaiah M, Venkat Reddy P, Ratna Reddy C, Malla Reddy A, Shankaraiha N, Lakshmi Narayan Reddy T, Pushpvall S and Pal Bhadra M, Eur J Med Chem., 2011, 46(9), 3820-3831; DOI:10.1016/j.ejmech.2011.05.050

6. Narender T, Venkateswarlu K, Vishnu Nayak B and Sarkar S, Tetrahedron Lett, 2011, 52(44), 5794-5798; DOI:10.1016/j.tetlet.2011.08.120

7. Rajakumar P and Raja S, Tetrahedron Lett., 2008, 49(46), 6539-6542; DOI:10.1016/j.tetlet.2008.09.005 
8. Suvitha S, Siddig Ibrahim A, Mohammed Ali M and Mohan S, Molecules, 2012, 17(6), 6179-6195; DOI:10.3390/molecules17066179

9. Ratchanok P, Amporn S, Prasit M, Chanin N, Supaluk, P, Somsak R and Virapong P, Eur J Med Chem., 2014, 85, 65-76; DOI:10.1016/j.ejmech.2014.07.087

10. Iqbal H, Prabhakar V, Sangita A, Chandrika B and Balasubramanium R, Med Chem Res., 2014, 23(10), 4383-4394; DOI:10.1007/s00044-014-1007-z

11. Manikdas and Kuntal Manna, J Toxicology, 2016, 1, 1-14.

12. Fathalla O A, Zaghary W A, Radwan H H, Awad S M and Mohamed S M, Archives Pharma Res., 2002, 25(3), 258-269; DOI:10.1007/BF02976623

13. Van Leeuwen R, Katlama, C, Kitchen V, Charles A B B, Roland T, McBride M, Didier I, Jonathan W, Andrew H, McDade H and Sven A D, Infectious Diseases, 1995, 171(5), 1166-1171; DOI:10.1093/infdis/171.5.1166

14. Owa T and Nagasu T, Experts Opinion Therapeutic Patents, 2000, 10(11), 17251740; DOI:10.1517/13543776.10.11.1725

15. Sapuran C T Scozzafava A and Casini A, Medicinal Research Reviews, 2003, 23(2), 146-189; DOI:10.1002/med.10025

16. Supuran C T and Scozzafava A, Immunology, Endocrine Metabolic Agents Medicinal Chemistry, 2001, 1(1), 61-97; DOI:10.2174/1568013013359131

17. Maren T H, Annual Review Pharmacology Toxicology, 1976, 16, 309-327; DOI:10.1146/annurev.pa.16.040176.001521

18. Boyd A E, Diabetes, 1998, 37, 847-850.

19. Thornber C W, Chem Soc Rev., 1979, 8, 563-580; DOI:10.1039/CS9790800563

20. Selvam P, Chandramohan M, De Clerk E, Witvrous M and Pannerouque C, European J Pharmaceutical Sci., 2001, 14(4), 313-316; DOI:10.1016/S0928-0987(01)00197-X

21. Park K H, Lee J W, Rayu Y B, Rayu H W and Lee S, Method for Screening Anticancer Compounds Containing Chalcone Compounds. 2014, CA2671987C.

22. Chun Wai Mai, Marzieh Yaeghoobi, Noorsaddah Abd Rahman, Kung Y B and Pichika M R, European J Med Chem., 2014, 77, 378-387; DOI:10.1016/j.ejmech.2014.03.002

23. Hamada N M M and Abdo N Y M, Molecules, 2015, 20(6), 10468-10486; DOI:10.3390/molecules200610468 